\title{
Article
}

\section{A blinded, randomized, controlled trial assessing conservative management strategies for frozen shoulder}

Russell, Sarah, Jariwala, Arpit, Conlon, Robert, Selfe, James, Richards, Jim and Walton, Michael

Available at http://clok.uclan.ac.uk/13737/

Russell, Sarah, Jariwala, Arpit, Conlon, Robert, Selfe, James, Richards, Jim ORCID: 0000-0002-4004-3115 and Walton, Michael (2014) A blinded, randomized, controlled trial assessing conservative management strategies for frozen shoulder. Journal of Shoulder and Elbow Surgery, 23 (4). pp. 500 507. ISSN 10582746

It is advisable to refer to the publisher's version if you intend to cite from the work. http://dx.doi.org/10.1016/j.jse.2013.12.026

For more information about UCLan's research in this area go to

http://www.uclan.ac.uk/researchgroups/ and search for <name of research Group>.

For information about Research generally at UCLan please go to http://www.uclan.ac.uk/research/

All outputs in CLoK are protected by Intellectual Property Rights law, including Copyright law. Copyright, IPR and Moral Rights for the works on this site are retained by the individual authors and/or other copyright owners. Terms and conditions for use of this material are defined in the policies page. 
Title

A Blinded, Randomised, Controlled Trial Assessing Conservative Management

Strategies for Frozen Shoulder

\section{Authors}

Russell SL BSc (Hons), MSc ${ }^{1}$

Selfe $\mathbf{J}^{2}$

Richards $\mathbf{J}^{3}$

Conlon $\mathrm{R}^{1}$

Walton MJ BMedSci, BMBS, MSc, FRCS(Tr\&Orth), MFSEM(UK) ${ }^{1}$

${ }^{1}$ Wrightington, Wigan and Leigh NHS Foundation Trust

${ }^{2,3}$ Allied Health Professions Research Unit, School of Sport, Tourism and The Outdoors.

University of Central Lancashire

\section{Correspondence address}

Sarah Russell

Physiotherapy Department,

Wrightington Hospital, Hall Lane, Apley Bridge, Wigan, WN6 9EP

Phone Number: 01257256534

Fax: 01257256279

Email: sarah.russell@wwl.nhs.uk 


\begin{abstract}
In order to assess current conservative physiotherapy strategies we assessed all primary care referrals for frozen shoulder to our physiotherapy department over a 12-month period. $17 \%$ of referrals met the criteria of a diagnosis of primary idiopathic frozen shoulder. 75 patients where randomly assigned one of three groups: group exercise class, individual multimodal physiotherapy and home exercises alone. All participants received education about frozen shoulder, advice on sleep, posture and pain relief. A single independent physiotherapist, who was blinded to the treatment groups, made all assessments. The Constant Score, Oxford Shoulder Score and SF-36 outcome measures were performed at baseline, six weeks, six months and one year. The Exercise Class lead to a significant improvement in shoulder symptoms and that this was greater than individual multimodal physiotherapy or home exercises alone with both Oxford and Constant scores. These findings were not demonstrated with the SF-36. This study demonstrates that a hospital based exercise class produces rapid recovery from a frozen shoulder with a minimum number of visits to the hospital and was more effective than individual physiotherapy or a home exercise programme. We would not recommend SF-36 as a valid patient reported outcome measure for the use in shoulder pathology.
\end{abstract}

Keywords Frozen shoulder, Physiotherapy, Adhesive capsulitis, Shoulder pain. 


\section{INTRODUCTION}

Frozen shoulder, or adhesive capsulitis, is defined as a condition of uncertain aetiology characterised by the spontaneous onset of pain with significant restriction of both active and passive range of movement of the shoulder ${ }^{1}$.

A primary or 'true' frozen shoulder occurs where there is no exogenous cause or preexisting condition. It presents an idiopathic decreased range of movement in which no systemic diagnosis, precipitating shoulder condition or radiographic explanation can be found ${ }^{2-4}$.

Despite considerable scientific research, the aetiology and pathology of frozen shoulder remain unknown ${ }^{5}$. The prevalence has been estimated at approximately $2-3 \%$ of adults in the general population ${ }^{6}$. However Bunker ${ }^{7}$ calculated a much smaller prevalence of $0.75 \%$ of the population based upon clinic attendance in secondary care. It usually develops between the ages of 40 and $70^{1,4,8}$ and rarely recurs in the same shoulder unless an injury or disease process predisposes the joint to repeat episodes of stiffness ${ }^{9,10}$.

Many studies have attempted to establish the most effective treatment for frozen shoulder but there still remains much debate in the literature. Currently there is no agreement on the standard management of this condition ${ }^{11}$. The controversy is due in part to a failure of many authors to precisely define and accurately identify frozen shoulder among other causes of shoulder pain and stiffness ${ }^{4,12}$. 
The Chartered Society of Physiotherapy has completed a project on the management of frozen shoulder ${ }^{13}$. Conclusions drawn from these evidence-based clinical guidelines suggest future researchers should report their physiotherapy interventions in sufficient detail to remove ambiguity consider multi-centre trials and focus on specific stages of frozen shoulder. In line with these recommendations, the aim of this study was investigate and report the clinical effectiveness of common physiotherapy interventions in the treatment of frozen shoulder using validate outcome measures to determine effectiveness. 


\section{METHODS}

Ethical approval was obtained from the Stockport Local Research Ethics Committee Clinical Trial Registration Number: 05/Q1401/86. All patients gave written informed consent before participating in the study,. The study used a randomised control trial (RCT) of three common physiotherapy interventions. Patients were randomly allocated to treatment groups and the study conformed to the CONSORT statement ${ }^{14}$.

Eligible patients were all new referrals to the physiotherapy with a diagnosis of frozen shoulder. Patients were assessed and inclusion and exclusion criteria verified (Table 1). Inclusion criteria were representative of the typical features of frozen shoulder ${ }^{15}$. The exclusion criteria served to eliminate patients with an inappropriate diagnosis of frozen shoulder and other inappropriate medical conditions complicating the pathology.

All patients underwent a standardised subjective and objective examination, as recommended by Wadsworth ${ }^{16}$ and Bowling et al. ${ }^{17}$. Routine anteroposterior and lateral radiographs were performed.

Following baseline evaluation, outcome measures were taken at six weeks, six months and at one year. All outcome measures were performed and recorded by an independent physiotherapist (SR) who was not involved in direct treatment of any patients and was blinded to the treatment allocation. Patients who expressed a desire to withdraw from the trial due to inability to cope with ongoing symptoms were recorded as having failed treatment and offered alternative treatment. 


\section{Intervention Groups}

Patients meeting the inclusion criteria and agreeing to participate in the study were then randomly allocated to one of the three treatment groups: group one: exercise class plus home exercises (Exercise Class), group two: individual multimodal physiotherapy plus home exercises (Multimodal Physiotherapy) and group three: home exercises alone (Home Exercise). These groups were identified to reflect current clinical practice. An independent statistician generated the assignment scheme using computer-generated permuted block randomisation. A random block length (chosen with equal probability from blocks of length six, nine or 12) was used.

The Exercise Class group treatment consisted of group therapy scheduled twice a week for six weeks. Patients performed a 50-minute exercise circuit comprising of 12 four-minute stations. Stations comprised of range of movement (ROM) and stretching exercises for all directions of shoulder movement. All patients were given careful instruction and demonstration of each exercise by a supervising physiotherapist. Exercise sheets were given to ensure compliance and aid understanding of the circuit.

The Multimodal group received two sessions of individual physiotherapy treatment per week for six weeks. The physiotherapist was a specialist in musculoskeletal physiotherapy with 11 years of sub-specialisation in shoulder therapy. The patients were also instructed on the specific shoulder exercises in the home exercise programme and given the information booklet. The treatment programme was based on local practise and expert opinion in the absence of clear consensus in the literature ${ }^{18}$. Treatment could be adjusted according to the severity of symptoms. It included Maitland mobilisations, which were progressed as the condition improved, soft tissue massage, myofascial trigger point release, 
heat, stretches and the identical home exercise programme as given to each of the other groups.

The Home Exercise group received instruction on the specific shoulder exercises in the information booklet.

All patients were given standardised advice and instructed in an identical home exercise programme. The information booklet included the home exercises, a description of frozen shoulder pathology, advice on sleep, posture and pain relief.

\section{Outcome Measures}

The primary outcome measure was the Constant-Murley Score ${ }^{19}$, which reflects shoulder function with accuracy, reliability, and reproducibility ${ }^{20,21,22}$. The score combines subjective and objective measures to produce a 100 point score, comprising four parameters: activities of daily living, range of motion, pain and strength. The minimal clinically important difference (MCID) is the smallest change in a score that patients perceive as meaningful, causing clinicians to consider a change in the patients' management ${ }^{23}$. There is no data, which clearly state the MCID for the Constant score. However, routine clinical practice within the organisations involved would normally consider a change of approximately 15 points to be clinically important.

The secondary outcome measures were the Oxford Shoulder Score and the SF-36. The OSS is a subjective questionnaire, which contains 12 questions derived from two parameters, pain and function. Scores from each of the questions are added to produce a single score ranging from 12 (least difficulties) to 60 (most difficulties) ${ }^{24}$. Patients complete the score unaided. The SF-36 is a widely used, self-administered, 36 item generic 
indicator used to assess general health ${ }^{25}$. It has recently been applied to the evaluation of shoulder disorders ${ }^{26}$. This is a questionnaire designed to assess eight dimensions of health status, which includes physical functioning (10 items), role limitations due to physical health problems (four items), bodily pain (two items), social functioning (two items), mental health (five items), role limitations due to emotional problems (three items), vitality and general health perceptions (five items).

\section{Statistical Analyses}

Data was analysed within groups to assess the effects of each intervention on the outcome measures and between groups, to compare the effects of the intervention. All data were tested to determine if normally distributed and where appropriate, a repeated measures one-way analysis of variance (RM ANOVA) on the outcome data was conducted. All data were tested using Mauchly's test for sphericity. Pairwise comparisons using the Least Squares Difference were conducted to investigate the differences between the different treatment groups and at the different time intervals following intervention. The baseline (pre-intervention) measurement was included as a covariate as it will be related to the repeated measurements following introduction of the different interventions rather than being an outcome of the intervention. The effect of the intervention (the average effect of the intervention over time) is then tested via the main effect of intervention group, whether the effect of the intervention varies over time is represented by the interaction between the intervention group and the repeated group and the repeated factor over time.

A power calculation was performed estimating the MCID of 15 points for the Constant Score, to achieve $80 \%$ power and 5\% significance. A cohort of 117 patients was required with 39 in each of the three treatment groups. 


\section{RESULTS}

A total number of 850 patients were referred to physiotherapy, with a primary care diagnosis of frozen shoulder, during a 12-month period. 705 did not fit the study inclusion criteria for primary idiopathic frozen shoulder. 70 patients declined to participate. Thus 75 patients entered the study and were randomly assigned to one of three groups (exercise class $n=25$, Multimodal physiotherapy $n=24$, home exercises $n=26$ ). One patient from the exercise class group died and was lost to follow-up. One patient in the multimodal physiotherapy group was referred for a steroid injection at 6 weeks and 2 from the home exercise group at 6 months, all were included in the analysis on an intention to treat basis.

The mean age was 51.1 years old (SD 6.84). The ratio of female to male was $1: 1.14$. The dominant arm was affected in $53 \%$ of the study population; $73 \%$ of patients were righthanded. The mean duration of symptoms was 5.79 months (SD 1.48). The primary analysis was intention-to-treat and involved all patients who were randomly assigned. There was no significant difference between the groups on baseline Constant or Oxford scores.

A repeated measures ANOVA demonstrated a significant difference in both Constant and Oxford scores between different time intervals $(\mathrm{p}<0.001)$. Further analysis using a pairwise comparison allowed comparison between the individual treatment groups. The patients in the exercise class group had a significantly better Constant score than either the multimodal physiotherapy or the home exercise groups $(\mathrm{p}<0.001, \mathrm{P}<0.001)$. This improvement was also demonstrated with the Oxford score $(\mathrm{p}=0.037, \mathrm{p}<0.001)$. The multimodal physiotherapy group showed significantly better Constant and Oxford scores than the home exercise group (Constant $\mathrm{p}=0.002$, Oxford $\mathrm{p}<0.001)$. 
A pairwise comparison showed a significant difference between six weeks and six months $(\mathrm{p}<0.001)$, six weeks and one year $(\mathrm{p}<0.001)$ and between six months and one year $(\mathrm{p}<0.001)$ for both the Constant and Oxford Scores. This demonstrates a continued improvement over time (Figures 1\&2).

Within the domains of the SF-36, bodily pain $(\mathrm{p}=0.011)$, mental health $(\mathrm{p}=0.009)$ and social function $(\mathrm{p}<0.001)$ all significantly improved over time on repeated measures ANOVA test. No other significant differences were found with either the repeated measures ANOVA. Pairwise comparisons did not show any significant differences between the treatment groups in any of the domains of the SF-36 outcome measure. 


\section{DISCUSSION}

Hanchard et al $^{13}$ report that the evidence for the management of frozen shoulder is inconclusive and is generally derived from studies with weak methodology. Kelly et al ${ }^{27}$ have suggested that there is no clear evidence to determine which patients may need formal supervised therapy as opposed to a home exercise programme. However, the findings of this study support and provide substantial evidence towards the use of an exercise class in the treatment of the patients with characteristics of, and a true diagnosis of frozen shoulder.

Clinically it is expected that a significant change in results would occur during the first six weeks due to the impact of treatment intervention. This is indeed reflected in the results of this study. Using an MCID for the Constant Score of 15 as a reference, $91 \%$ of patients in the exercise group demonstrated an improvement greater than the MCID, similarly in the individual multimodal physiotherapy group by $68 \%$ and in the home exercise group by $41 \%$.

The results of the study confirmed that patients seen in an exercise class and supervised by a physiotherapist had better outcomes and recovered in a shorter time frame than those patients in an individual multimodal physiotherapy or home exercise programme. This can influence clinical practice by potentially reducing the number of individual physiotherapy treatment sessions, which increases cost effectiveness, as suggested by Carr et al ${ }^{28}$. It may also improve care pathways by initiating effective management from initial diagnosis. This could standardise treatment outcomes and impact upon the need for surgical or more invasive interventions. 
This however, is in contrast to the work by Levine et al ${ }^{29}$ who suggested that patients placed on a therapist directed home exercise programme had the same outcomes at short and long term follow-ups as those treated with other interventions. Kivimaki et al ${ }^{30}$ compared patients treated with a home exercise programme to those with manipulation under anaesthetic and a home exercise programme. Other than a slight increase in ROM, the group performing just a home exercise programme did not differ at any follow up in pain or working ability.

The significant improvements seen in this study may be due to the benefits of group exercise. Group exercise classes provide a clinical setting in which patients can discuss their condition with others who are in a similar position. This may reassure patients and provide them with peer support and the motivation they need to continue and progress their rehabilitation. Patients were taught self-management of their condition and how to deal with any increase in pain. Behavioural changes during the treatment period, relating to improvement of self-management could reduce the utilisation of health care services during the follow up period and reduce sick leave in patients. Further work into the psychological effects of conditions and the benefits of group therapies are an important avenue for further research.

One of the interesting findings of this study relates to the diagnosis of frozen shoulder. Only $17 \%$ (145/850) of initial referrals from primary care met the inclusion criteria for primary idiopathic frozen shoulder. This suggests a need to educate primary care physicians and physiotherapists involved in the diagnosis and management of frozen shoulder to improve their clinical diagnostic accuracy. This low number of "true frozen shoulders" in the population questions the estimations of primary care prevalence ${ }^{6}$ and 
more in keeping with the estimations made by Bunker ${ }^{7}$. This difference in true prevalence made recruitment much slower than we had anticipated and lead to the key limitation of this study in that the number of patients recruited did not meet our initial power calculations. However despite the smaller numbers we have demonstrated statistically significant differences between the treatment modalities studied.

A further limitation of the study is the absence of a natural history control group. The constraints of the ethics process prevented a no treatment arm being included in the study. However, the home exercises group represents a control against direct physiotherapy management and may well represent a close proximity to the natural history.

This study has also provided information regarding the appropriate use of outcome measures. Both the Constant Score ${ }^{19,20,21,22}$ and Oxford Score ${ }^{24,31}$ have been validated for the assessment of shoulder conditions. Both of these scoring systems have shown significant benefits of physiotherapy intervention in this study. The SF-36 however showed very few significant differences overall and none between the groups. This lack of sensitivity of the SF-36 in the assessment of shoulder pathology is also reported by Carette et al ${ }^{32}$, who found no significant differences between the groups they had analysed in their study comparing the use of corticosteroid injection, a supervised exercise programme and a combination of the two and placebo in the treatment of frozen shoulder. Beaton and Richards ${ }^{33}$ concluded that the SF-36 was not sensitive enough to detect the disability experienced by patients with upper extremity problems. Griggs et al ${ }^{34}$ used the SF-36 in a study evaluating the efficacy of a specific four-direction shoulder-stretching exercise programme. They concluded that the SF-36 did not demonstrate significantly lower scores for the satisfied patients compared with the general population. Buckbinder et $\mathrm{al}^{35}$, in a previous trial of oral steroids for frozen shoulder, discovered that only the bodily pain 
subscale of the SF-36 detected a benefit of prednisolone over placebo at three weeks, despite large clinically significant benefits observed for other outcomes including pain, function and ROM.

In conclusion, this study demonstrates that an exercise class, aimed at a rapid recovery rate with a minimum number of interventions, provides superior outcomes in relieving the signs and symptoms of frozen shoulder compared to those having individual multimodal physiotherapy or performing home exercises. However, standard multimodal physiotherapy remains a good alternative and has been demonstrated to be significantly better than unsupervised exercise at home. We would recommend the use of the Oxford or Constant Score outcome measures but would advise against the use of the SF-36 as it does not appear to be a sensitive reflection of shoulder pathology. We have highlighted the poor level of diagnostic accuracy in referrals and emphasis the need for better education for primary care physicians and physiotherapists in the assessment of shoulder pathology. In the current climate of greater emphasis being placed on referral management, care in the community and primary care triage, this has become more important than ever. 


\section{REFERENCES}

1. Reeves B. The natural history of the frozen shoulder syndrome. Scandinavian Journal of Rheumatology 1975;4:193-6.

2. Neviaser RJ, Neviaser TJ. The frozen shoulder: diagnosis and management. Clinical Orthopaedics 1987;223:59-64.

3. Kelly IG. Frozen shoulder. In: Kelly IG. The practice of shoulder surgery. Oxford: Butterworth-Heinnemann 1993;196-205.

4. Stam H. Frozen Shoulder: A review of current concepts. Physiotherapy 1994;80:588-99.

5. Hannafin JA, Chiaia TA. Adhesive capsulitis: a treatment approach. Clinical Orthopaedics 2000;372: 95-109.

6. Lubiecki M , Carr A. Frozen shoulder: past, present and future. Journal of Orthopaedic Surgery 2007;15 (1).

7. Bunker TD. Time for a new name for frozen shoulder-contracture of the shoulder. Shoulder and Elbow 2009;1:4-9.

8. Binder AI, Bulgen DY, Hazleman BL, Parr G, Roberts S. A controlled study of oral prednisolone in frozen shoulder. British Journal of Rheumatology 1986;25:288-92. 
9. Di Fabio, RP. Secrets of diagnosis. Journal of Orthopaedic Sports Physical Therapy 1998;29:504.

10. Hand C, Athanason N, Matthews T, Carr A. Pathology of frozen shoulder. Journal of Bone and Joint Surgery 2007;89:928-32.

11. Dundar U, Toktas H, Cakir T, Evcik D, Kavuncu V. Continuous passive motion provides good pain control in patients with adhesive capsulitis. International Journal of Rehabilitation Research 2009;32(3):193-198.

12. Green S, Buckbinder R, Hetrick S. Physiotherapy interventions for shoulder pain. Physiotherapy 2009;89:335-6.

13. Hanchard N, Goodchild L, Thompson J, O’Brien T, Richardson C, Davison D, Watson H, Wragg M, Mtopo S, Scott M. Evidence-based clinical guidelines for the diagnosis, assessment and physiotherapy management of contracted (frozen) shoulder." v.1.2, 'standard' physiotherapy. Endorsed by the Chartered Society of Physiotherapy 2011. Available at: www.csp.org.uk/skipp

14. Altman DG, Schulz KF, Moher D, Egger M, Davidoff F, Elbourne D, Gotzsche P, Lang T. The revised consort statement for reporting randomised trials: explanation and elaboration. Annals of Internal Medicine 2001;134:663-94.

15. Miller MD, Wirth MA, Rockwood CA. Thawing the frozen shoulder: the "patient". Clinical Orthopaedics 1998;19:849-53. 
16. Wadsworth CT. Frozen shoulder. Physical Therapy 1986;66:1878-83.

17. Bowling RW, Rochar PA, Erhard R. Examination of the shoulder complex. Physical Therapy 1986;66:1866-78.

18. Ryans I, Montgomery A, Galway R, Kernohan WG, McKane R. A randomised controlled trial of intra-articular triamcinolone and/or physiotherapy in shoulder capsulitis. Rheumatology 2005;44:529-35

19. Constant CR, Murley AGH. A clinical method of functional assessment of the shoulder. Clinical Orthopaedics 1987;214:160-4.

20. Gazielly F, Gleyze P, Matangnon C. Functional and anatomical results after rotator cuff repair. Clinical Orthopaedics and Related Research 1994;4:43-53.

21. Bankes MJK, Crossman JE, Emery RJH. A standard method of shoulder strength assessment for the Constant Score with a spring balance. Journal of Shoulder and Elbow Surgery 1998;7:116-21.

22. Yian EH, Ramappa AJ, Arneberg O, Gerber C. The Constant Score in normal shoulders. Journal of Shoulder and Elbow Surgery 2005;14:128-33.

23. Fayers $P$, Machin, D. Quality of Life: The assessment, analysis and reporting of patient-reported outcomes 2nd edition. Wiley, New York. 2007. 
24. Dawson J, Fitzpatrick R, Carr A. Questionnaire on the perceptions of patients about shoulder surgery. Journal of Bone and Joint Surgery (Br) 1996;78:593-600.

25. Ware J, Sherbourne CD. The MOS 36-item Short Form Health Survey (SF36). Medical Care 1992;30:473-81.

26. Smith KL, Harryman DT, Antoniou J, Campbell B, Sidles JA, Matsen FA. A prospective, multipractice study of shoulder function and health status in patients with documented rotator cuff tears.” Journal of Shoulder and Elbow Surgery 2000;9(5):395-402.

27. Kelly MJ, McClure PW, Leggin BG. Frozen Shoulder: Evidence and a Proposed Model Guiding Rehabilitation. Journal of Orthopaedic and Sports Physical Medicine 2009;39:135-48.

28. Carr JL, Klaber Moffett JA, Howarth E, Richmond SJ, Torgerson DJ, Jackson DA, Metcalfe CJ. A randomized trial comparing a group exercise programme for back pain patients with individual physiotherapy in a severely deprived area. Disability and Rehabilitation 2005;27:16 929-937.

29. Levine WN, Kashyap CP, Bak SF, Ahmad CS, Blaine TA, Bigliani LU. Nonoperative management of idiopathic adhesive capsulitis. Journal of Shoulder and Elbow Surgery 2007;16:569-73.

30. Kivimaki J, Pohjolainen T, Malmivaara A, Kannisto M, Guillaume J, Seitsalo, 
Nissinen M (2007) "Manipulation under anaesthesia with home exercises versus home exercises alone in the treatment of frozen shoulder: A randomized, controlled trial with 125 patients.” Journal of Shoulder and Elbow Surgery 2007;16:722-6.

31. Othman A, Taylor G. Is the Constant Score reliable in assessing patients with frozen shoulder. 60 shoulders scored 3 years after manipulation under anaesthetic. Acta Orthopaedica Scandinavica 2004;75:114-6.

32. Carette S, Moffett H, Tardif J, Bessette L, Morin F, Fremont P, Bykerk V, Thorne C, Bell M, Bensen W, Blanchette C. Intrarticular Corticosteroids, supervised physiotherapy, or a combination of the two in the treatment of adhesive capsulitis of the shoulder: a placebo-controlled trial. Arthritis Rheumatology 2003;48:829-38.

33. Beaton DE, Richards RR. Measuring function of the shoulder. A crosssectional comparison of five questionnaires. Journal of Bone and Joint Surgery. 1996;78:882-90.

34. Griggs SM, Ahn A, Green A. Idiopathic adhesive capsulitis: a prospective functional outcome study of non-operative treatment. Journal of Bone and Joint Surgery (Am) 2000;82:1398-1407.

35. Buckbinder R, Hoving JL, Green S, Hall S, Forbes A, Nash P. Short course prednisolone for adhesive capsulitis (frozen shoulder or stiff painful shoulder): a randomised, double blind, placebocontrolled trial. Annals of Rheumatic Diseases 2004;63:1460-9. 


\section{Table 1}

\section{Inclusion and Exclusion Criteria}

\section{INCLUSION CRITERIA}

Age 40 to 70 years old

Patients reported local shoulder pain, frequently present over either the anteromedial aspect of the shoulder extending distally into the biceps region, or over the lateral aspect of the shoulder extending into the lateral deltoid region. Symptoms present for at least three months.

Spontaneous onset of a painful stiff shoulder

Marked loss of active and passive global shoulder motion, with at least $50 \%$ loss of external rotation

Normal x-rays on anteroposterior and axillary radiographs of the glenohumeral joint

\section{EXCLUSION CRITERIA}

Radiographic pathological findings or glenohumeral osteoarthritis on x-ray

Clinical evidence of significant cervical spine disease

History of significant trauma to the shoulder

Local corticosteroid injection or any physiotherapy intervention to the affected shoulder within the last three months

Cerebral vascular accident affecting the shoulder

Inflammatory joint disease affecting the shoulder

Bilateral frozen shoulder due to possible underlying systemic cause 
Thyroid disease

Any coronary event, post coronary artery by-pass or catheterisation prior to the clinical appearance of frozen shoulder

Prior surgery, dislocation or fractures on the affected shoulder

Active medico legal involvement 